\title{
Common Birds of Chitwan National Park, Nepal
}

\author{
Parveen Kumar Jha ${ }^{1}$ \\ ${ }^{1} \mathrm{Ph} . \mathrm{D}$ Scholar, Mewar University \\ Gangrar, Chittorgar Rajasthan India
}

\begin{abstract}
This research paper gives checklist of common birds of Chitwan National Park, which is a wildlife protected area in south-central Nepal. It covers tropical and sub-tropical vegetation. It is first protected area and includes $932 \mathrm{sq}$. km. Common birds observed are about 170 belonging to 48 Avian families during 2013-2014. Present investigator has very minutely observed birds in habitat conditions. Bird species were recognized by very high binocular. Birds were thoroughly studied from point of view of Taxonomy. Machans were also erected for observing birds
\end{abstract}

KEY WORDS: Machan, Taxonomy, Strata

\section{INTRODUCTION}

Chitwan National Park $\left(27^{\circ} 30^{\prime} \mathrm{N}, 8^{\circ} 20^{\prime} \mathrm{E}\right)$ was established in 1973 with the beginning of conservation of biodiversity in Nepal. Chitwan National Park covers an area of 932 sq. km. and is located in south-central Nepal. In 1973, the National Parks and Wildlife Conservation Act was enacted and Chitwan National Park was declared the first national park of Nepal (DNPWC, 2012). By 1977, the promulgated boundaries were increased from $540 \mathrm{sq}$. km. to $932 \mathrm{sq} . \mathrm{km}$. Recognizing its unique ecosystems of international significance; UNESCO declared the park as a World Heritage Site in 1984 and is also identified as an important bird area (IBA) by Bird Life International. In 1996, an area of $750 \mathrm{sq}$. km. surrounding the park was declared a buffer zone, which consists of forests and private lands. Bishajari and associated lakes in the buffer zone of the Chitwan National Park were declared wetlands of international importance under the Ramsar Convention in 2003 (DNPWC, 2012).

Extended in four districts of Central Terai, this national park includes the area of Chitwan 74\%; Parsa 15\%; Makwanpur 7\% and Nawalparasi 4\%. It is bordered by Parsa Wildlife Reserve (499

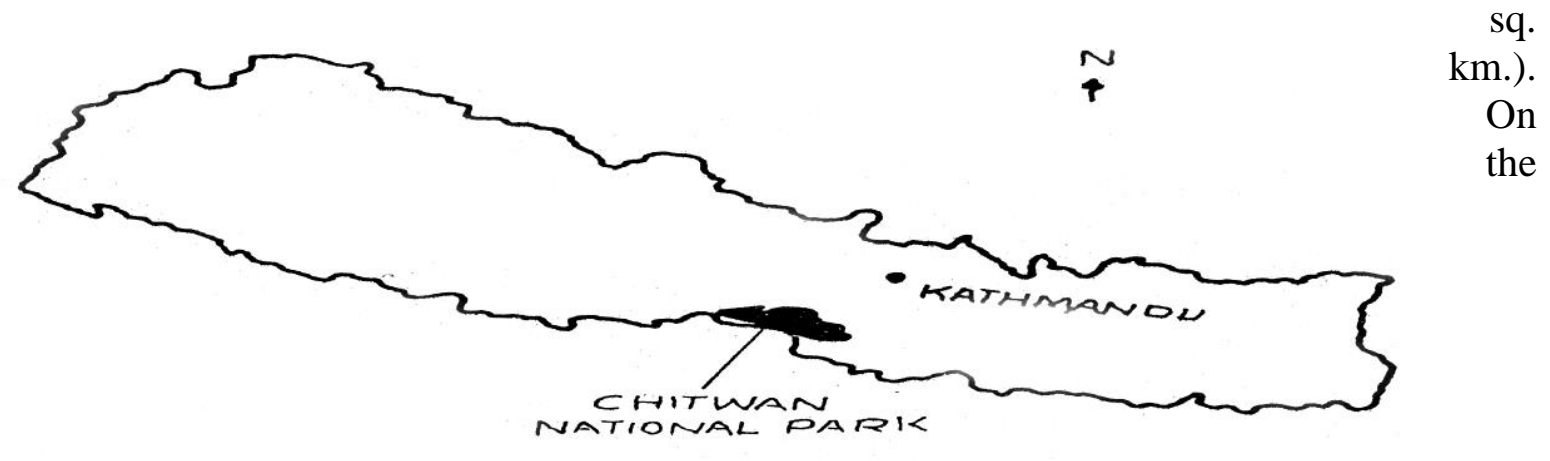

eastern side and on all other river sides make natural boundary for the park (DNPWC, 2012). 
Fig No 1: Location map of Chitwan National Park

The Chitwan National Park consists of tropical and subtropical forests with mostly Sal (Shorea robusta) forests. Sal forest covers $70 \%$ of the park, tall grasslands $15 \%$ and remaining by riverine and other forest types (Dinerstein, 2003). The Chitwan National Park has many rivers and lakes. Chitwan National Park is the third destination of tourists in Nepal (Office of Chitwan National Park, 2012).

The present author undertook deep and exhaustive studies on avian biodiversity of birds of Chitwan National Park during different seasons of 2013-2014. The study observed about 400 birds in Natural habitat in different seasons at different localities of the park. He noted distribution and identification of birds very carefully and minutely.

\section{HISTORICAL REVIEW}

Flemings, et. al. (1979) described in their pioneer book on Birds of Nepal, distribution of birds in Chitwan National Park. Similarly, Inskip, et. al. (2000) also have given locality of birds with reference to Chitwan National Park.Bird Education Society: Sauraha has also published a bird checklist of Chitwan. In the first edition of the book 401 species of birds are described. In the second edition, 590 species representing 59 families have been included in the checklist while in the recent edition (2014), about 625 species have been listed. Baral, et. al. (2006) published a checklist of birds of Chitwan. About 35 globally threatened birds have been seen in Chitwan National Park. (Baral and Inskipp, 2005). Recently, Shakya (2014) has written an illustrated book on Birds of Chitwan.

\section{STRATA OF SITE}

The present investigator carried out his investigation in 4 stratas during different seasons of the year 2013-2014. These stratas are based on vegetation and habitats. Surveys were conducted from sunrise to noon under favourable conditions.

Strata 1: Balkmiki Ashram to Temple Tiger

Strata 2: Temple Tiger to Kasara

Strata 3: Kasara to Sauraha

Strata 4: Sauraha to Sunachari

\section{MATERIAL AND METHODS}

Different bird species have been observed in natural habitat during trips by using powerful binoculars and telescope 20 x 60 Nikkon Coy. Japan of Yashika and Ashai Pentex. For photography digital camera of Nikkon $400 \mathrm{x}$ tele lens was used. Various secondary data were also collected. Migratory birds were also noted.

\section{RESULTS}


The present investigator observed about 400 birds. Some birds reported by earlier researchers were not seen even after extensive survey.

\section{COMMON BIRDS OF CHITWAN NATIONAL PARK \\ PHASIANIDAE}

Francolinus francolinus, Black Francolin

Gallus gallus, Red Junglefowl

Lophura leucomelanos, Kalij Pheasant

Pavo cristatus, Indian Peafowl

DENDROCYGNIDAE

Dendrocygna javanica, Lesser Whistling Duck

ANATIDAE

Tadorna ferruginea, Ruddy Shelduck

Anas strepera, Gadwall

Anas penelope, Eurasian wigeon

Anas platyrhynchos, Mallard

Rhodonessa rufina, Red-crested Pochard

Mergus merganser, Common Merganser

PICIDAE

Dendrocopos canicapillus, Grey-capped Pygmy Woodpecker

Dendrocopos macei, Fulvous-breasted Woodpecker

Picus chlorolophus, Lesser Yellownape

Picus xanthropygaeus, Streak-throated Woodpecker

Picus canus, Grey-headed Woodpecker

Dinopium shorii, Himalayan Flameback

Dinopium benghalense, Black-rumped Flameback

Chrysocolaptes lucidus, Greater Flameback

MEGALAIMIDAE

Megalaima lineata, Lineated Barbet

Megalaima haemacephala, Coppersmith Barbet

BUCEROTIDAE

Anthracoceros albirostris, Oriented Pied Hornbill

UPUPIDAE

Upupa epops, Common Hoopoe

TROGONIDAE

Horpactes erythrocephalus, Red-headed Trogon

CORACIIDAE

Coracias benghalensis, Indian Roller

Eurystomus orientalis, Dollarbird

MEROPIDAE

Merops orientalis, Green Bee-eater

Merops philippinus, Blue-tailed Bee-eater

Merops leschenaulti, Chestnut-headed Bee-eater

ALCEDINIDAE

Alcedo atthis, Common Kingfisher

HALCYONIDAE 
Halcyon capensis, Stork-billed Kingfisher

Halcyon smyrnensis, White-throated Kingfisher

CERYLIDAE

Ceryle rudis, Pied Kingfisher

\section{CUCULIDAE}

Hierococcyx varius, Common Hawk Cuckoo

Cuculus micropterus, Indian Cuckoo

Cuculus canorus, Eurasian Cuckoo

Eudynamy scolopacea, Asian Koel

Phaenicophaeus tristis, Green-billed Malkoha

CENTROPODIDAE

Centropus sinensis, Greater Coucal

Centropus bengalensis, Lesser Coucal

PSITTACIDAE

Psittacula eupatria, Alexandrine Parakeet

Psittacula krameri, Rose-ringed Parakeet

Psittacula himalayana, Slaty-headed Parakeet

Psittacula cyanocephala, Plum-headed Parakeet

Psittacula alexandri, Red-breasted Parakeet

APODIDAE

Zoonavena sylvatica, White-rumped Needletail

Apus affinis, House Swift

HEMIPROCNIDAE

Hemiprocne coronata, Crested Treeswift

STRIGIDAE

Ninox scutulata, Brown Hawk Owl

Glaucidium radiatum, Jungle Owlet

Athene brama, Spotted Owlet

CAPRIMULGIDAE

Caprimulgus macrurus, Large-tailed Nightjar

Caprimulgus affinis, Savanna Nightjar

COLUMBIDAE

Columba livia, Rock Pigeon

Streptopelia chinensis, Spotted Dove

Streptopelia tranquebarica, Red-collared Dove

Streptopelia decaocto, Eurasian Collared Dove

Chalcophaps indica, Emerald Dove

Treron bicincta, Orange-breasted Green Pigeon

Treron pompadora, Pompadour GreenPigeon

Treron phoenicoptera, Yellow-footed Green Pigeon

RALLIDAE

Amaurornis akool, Brown Crake

Amaurornis phoenicurus, White-breasted Waterhen

Gallinula chloropus, Common Moorhen

SCOLOPACIDAE

Gallinago gallinago, Common Snipe 
Tringa nebularia, Common Greenshank

Tringa ochropus, Green Sandpiper

Actitis hypoleucos, Common sandpiper

Calidris temminckii, Temminck's Stint

JACANIDAE

Metopidius indicus, Bronze-winged Jacana

CHARADRIIDAE

Charadrius dubius, Little-Ringed Plover

Vanellus duvaucelii, River Lapwing

Vanellus indicus, Red-wattled Lapwing

ACCIPITRIDAE

Pandion haliaetus, Osprey

Milvus migrans, Black Kite

Ichthyophaga ichthyaetus, Grey-headed Fish Eagle

Spilornis cheela, Crested Serpent Eagle

Accipiter badius, Shikra

Pernis ptilorhynncus, Oriental Honey-buzzard

FALCONIDAE

Falco tinnunculus, Common Kestrel

ANHINGIDAE

Anhinga melanogaster, Darter

PHALACROCORACIDAE

Phalacrocorax carbo, Great Cormorant

ARDEIDAE

Egretta garzetta, Little Egret

Mesophoyx intermedia, Intermediate Egret

Bubulcus ibis, Cattle Egret

Ardeola grayii, Indian Pond Heron

Butorides striatus, Little Heron

Nycticorax nycticorax, Black-crowned Night Heron

THRESKIORNITHIDAE

Pseudibis papillosa, Black Ibis

CICONIIDAE

Anastomus oscitans, Asian Openbill

Ciconia episcopus, Wooly-necked Stork

Ciconia nigra, Black Stork

Leptoptilos Javanicus, Lesser Adjutant

PITTIDAE

Pitta sordida, Hooded Pitta

Pitta brachyura, Indian Pitta

IRENIDAE

Chloropsis aurifrons, Golden-fronted Leafbird

Chloropsis hardwickii, Orange-bellied Leafbird

LANIIDAE

Lanius cristatus, Brown Shrike

Lanius schach, Long-tailed Shrike 
Lanius tephronotus, Grey-backed Shrike CORVIDAE

Urocissa erythrorhyncha, Red-billed Blue Magpie

Dendrocitta vagabunda, Rufous Treepie

Corvus splendens, House Crow

Corvus macrorhynchos, Large-billed Crow

Artamus fuscus, Ashy Woodswallow

Oriolus oriolus, Eurasian Golden Oriole

Oriolus xanthornus, Black-hooded Oriole

Coracina macei, Large Cuckooshrike

Pericrocotus cinnamomeus, Small Minivet

Pericrocotus flammeus, Scarlet Minivet

Hemipus picatus, Bar-winged Flycatcher-Shrike

Rhipidura albicollis, White-throated Fantail

Dicrurus macrocercus, Black-Drongo

Dicrurus caerulescens, White-bellied Drongo

Dicrurus hottentottus, Spangled Drongo

Terpsiphone paradisi, Asian Paradise-flycatcher

Aegithina tiphia, Common Iora

Tephrodornis pondicerianus, Common Woodshrike

MUSCICAPIDAE

Myophonus caeruleus, Bluewhistling Thrush

Ficedula parva, Red-throated Flycatcher

Culicicapa ceylonensis, Grey-headed Canary Flycatcher

Cyornis poliogenys, Pale-chinned Flycatcher

Luscinia calliope, Siberian Rubythroat

Copsychus saualaris, Oriental Magpie Robin

Copsychus malabaricus, White-rumped Shama

Saxicola torquata, Common Stonechat

Saxicola leucura, White-tailed Stonechat

Saxicola caprata, Pied Bushchat

STURNIDAE

Sturnus malabaricus, Chestnut-tailed Starling

Sturnus contra, Asian Pied Starling

Acridotheres tristis, Common Myna

Acridotheres fuscus, Jungle Myna

SITTIDAE

Sitta castanea, Chestnut-bellied Nuthatch

Sitta frontalis, Velvet-fronted Nuthatch

PARIDAE

Parus major, Great Tit

HIRUNDINIDAE

Riparia paludicola, Plain Martin

Hirundo rustica, Barn Swallow

Hirundo daurica, Red-rumped Swallow

PYCNONOTIDAE 
Pycnonotus jocosus, Red-whiskered Bulbul

Pycnonotus leucogenys, Himalayan Bulbul

Pycnonotus cafer, Red-vented Bulbul

CISTICOLIDAE

Prinia hodgsonii, Grey-breasted Prinia

Prinia flaviventris, Yellow-bellied Prinia

Prinia inornata, Plain Prinia

Cisticola juncidis, Zitting Cisticola

ZOSTEROPIDAE

Zosterops palpebrosus, Oriental White-eye

SYLVIIDAE

Orthotomus sutorius, Common Tailorbird

Acrocephalus dumetorum, Blyth's Reed Warbler

Phylloscopus collybita, Common Chiffchaff

Phylloscopus reguloides, Blyth's Leaf Warbler

Phylloscopus trochiloides, Greenish Warbler

Garrulax leucolophus, White-crested Laughingthrush

Garrulax monileger, LesserNecklaced Laughingthrush

Garrulax pectoralis, Greater Necklaced Laughingthrush

Pellorneum ruficeps, Puff-throated Babbler

Macronous gularis, Striped Tit Babbler

Timalia pileata, Chestnut-capped Babbler

Turdoides earlei, Striated Babbler

Turdoides striatus, Jungle Babbler

ALAUDIDAE

Mirafra assamica, Rufous winged Bushlark

Calandrella raytal, Sand Lark

NECTARINIIDAE

Aethopyga siparaja, Crimson Sunbird

PASSERIDAE

Passer domesticus, House Sparrow

Passer montanus, Eurasian Tree Sparrow

Motacilla maderaspatensis, White-browed Wagtail

Anthus rufulus, Paddyfield Pipit

Anthus hodgsoni, Olive-backed Pipit

Anthus roseatus, Rosy Pipit

Ploceus philippinus, Baya Weaver

Lonchura punctulata, Scaly-breasted Munia

Common Birds $=170$, which include birds of forest, grassland and wetlands.

Families -48

\section{DISCUSSION}

Salim Ali and Dillon Ripley (1987) have described Birds of Nepal in their extensive work. Flemings et. al., Richard Grimmett, Carol Inskipp and Tim Inskipp have also mentioned Birds of Chitwan. Hem Sagar Baral and Gopal Prasad Upadhyay give checklist of Birds of Chitwan.

However, the present investigator has studied characters of common birds of Chitwan National Park. Considering abundance of species, fairly common, occasional, uncommon, rare and very 
unlikely species. Besides, it has been noted that the seasons effect greatly on breeding and migration of Chitwan Aves. The present investigator has investigated 4 stratas during different seasons of the year 2013-2014. He reported in this paper 171 common birds of the 47 families. It has also been found on computation about species richness is as follows: Corvidae (18), Sylviidae (13), Muscicapidae (10), Passeridae (8), Columbidae (8), Picidae (8) and Phasianidae (5).

\section{CONCLUSION}

The investigator provides common list of the avian fauna of Chitwan National Park which is very significant National Park of Central Nepal, Terai and out of 851 Nepal birds more than 500 are found at this site. Data provide information on seasonal changes. It will also indicate various problems which avian fauna is facing and its solutions. Results obtained from this research will provide valuable reference for future researchers.

\section{ACKNOWLEDGEMENTS}

The present investigator expresses deep gratitude to Mewar University Chittorgarh for providing an opportunity to work on the project. He is also highly indebted to Prof. Dr. T.C. Majupuria for supervision and guidance in this research work. Thanks are also due to Dr. Tatwa P. Timisina for his inestimable help in this research work. Without kind guidance of Dr. Tatwa, this research work could have not been completed. I am also obliged to Dr. Hem Sagar Baral, an eminent ornithologist for his valuable suggestions and equally thankful to his wife Ms. Kanchan Jha for encouragement and support during this work.

\section{BIBLIOGRAPHY}

Ali, S. and Ripley, S.D. (1987). Handbook of Birds of India and Pakistan-1968 - 1974: Revised Edition Compact Handbook. Bombay: Oxford University Press. Vol. 10.

Baral, H.S. and Upadhyay, Birds of Chitwan (4th Edition). (2006). Department of National Parks and Wildlife Conservation and Bird Conservation. Kathmandu: Nepal.

Baral, H.S., and Inskipp, C. (2005). Important Bird Areas in Nepal: Bird Conservation Nepal and Bird Life International. Kathmandu and Cambridge.

Bird Checklist Chitwan. Bird Education Society. Sauraha: Chitwan.

Dinerstein, E. (2003). The return of the Unicorns: the natural history and conservation of the greater one-horned rhinoceros. New York: Columbia Press. 316

Fleming, R.L. Sr. Fleming R.L. Jr. and Bangdel, L.S., Birds of Nepal. (1979). Kathmandu. Avalok Publsihers.

Inskipp. C. and Inskipp. T. (2000). A guide to birds of Nepal. London: U.K. Crown Helm.

Nepal Protected Areas. (2014). Kathmandu. Department of National Parks and Wildlife Conservation.

Shakya, B. and Suresh. (2014). A Photographic Guide to the Birds of Chitwan. Kathmandu. 